\title{
Characterization of ion track morphology formed by swift heavy ion irradia- tion in silicon oxynitride films
}

\author{
P. Mota-Santiago ${ }^{1, a}$, D. Schauries ${ }^{1}$, A. Nadzri ${ }^{1}$, K. Vora ${ }^{2}$, M. C. Ridgway ${ }^{1}$, and P. Kluth ${ }^{1}$ \\ ${ }^{1}$ Department of Electronic Materials Engineering, Australian National University, Canberra ACT 2601, Australia \\ ${ }^{2}$ Australian National Fabrication Facility, ACT Node, Research School of Physics and Engineering, Australian National University, \\ Canberra ACT 2601, Australia
}

\begin{abstract}
Amorphous silicon oxynitride $\left(\mathrm{SiO}_{x} \mathrm{~N}_{y}\right)$ possess interesting optical and mechanical properties. Here, we present direct evidence for the formation of ion tracks in $1 \mu \mathrm{m}$ thick silicon oxynitride of different stoichiometries. The tracks were created by irradiation with $185 \mathrm{MeV} \mathrm{Au}^{13+}$ ions. The samples were studied using spectral reflectometry and Rutherford backscattering spectrometry (RBS), with the track morphology characterised by means of small angle X-ray scattering (SAXS). The radial density of the ion tracks resembles a core-shell structure with a typical radius of $\sim 1.8+2.4 \mathrm{~nm}$ in the case of $\mathrm{Si}_{3} \mathrm{~N}_{4}$ and $2.3+3.2 \mathrm{~nm}$ for $\mathrm{SiO}_{2}$.
\end{abstract}

\section{Introduction}

Swift heavy ions (SHI) interact predominantly with matter via electronic excitations and subsequently, the energy is transferred to the lattice where it can yield melting along the ion path. While in crystalline materials the rapid quenching can freeze in an amorphous track with a well-defined cylindrical structure, in amorphous materials a more complex process takes place.

The energy deposition of high energy ions proceeds by inelastic collisions between the energetic ion and the electronic cloud around the lattice (electronic stopping power). As a result, a very narrow and highly ionised region is produced [1]. The ionized electrons can loose their energy by colliding with outer electrons (electronic thermal conductivity) or by interacting with the lattice via electronphonon coupling [2]. The energy transferred to the lattice locally increases the temperature of the material leading to a cylindrical molten region of a few nanometers radius, if the electronic stopping power is sufficient.

The structure of the ion tracks can generally be observed by transmission electron microscopy (TEM) in a crystalline material, while direct observation in amorphous materials is difficult due to a very low contrast between the track region and the rest of the material [3]. In this work, small angle X-ray scattering (SAXS) is employed to study the fine structure of ion tracks in silicon oxynitride of different stoichiometries. This technique is sensitive to small density variations and averages over a large number of ion tracks.

Due to the high tunability in their optical and mechanical properties, silicon oxynitrides have been attracting a lot of attention for gradient-index optics [4], as antireflection coating and for surface passivation of crystalline

\footnotetext{
ae-mail: pablo.mota@anu.edu.au
}

Table 1: Chemistry used during the chemical vapour deposition of the silicon oxynitride films (values in sccm) and thickness in nanometers measured via spectral reflectometry; the uncertainty in thickness is less than $0.5 \mathrm{~nm}$.

\begin{tabular}{cccccc} 
& $\mathrm{Si}_{3} \mathrm{~N}_{4}$ & $\# 1$ & $\# 2$ & $\# 3$ & $\mathrm{SiO}_{2}$ \\
\hline & & & & & \\
$\mathrm{SiH}_{4}$ & 8 & 8 & 8 & 8 & 8 \\
$\mathrm{NH}_{3}$ & 14 & 12 & 10.5 & 5.6 & 0 \\
$\mathrm{~N}_{2}$ & 980 & 910 & 790 & 525 & 270 \\
$\mathrm{~N}_{2} \mathrm{O}$ & 0 & 70 & 190 & 455 & 710 \\
Thickness & 1180 & 1012 & 968 & 990 & 939
\end{tabular}

silicon solar cells [5] or for the new generation of lightemitting devices [6], making them suitable candidates for the synthesis of nanostructures by ion irradiation [3].

In this work we present direct measurements of ion tracks in silicon oxynitride formed by SHI irradiation and characterize their morphology.

\section{Experimental}

Silicon oxynitride films were deposited at the Australian National Fabrication Facility (ANFF) on c-Si(100) wafers with an Oxford Plasma Technology 100PECVD system. The $\mathrm{SiH}_{4}: \mathrm{NH}_{3}: \mathrm{N}_{2}: \mathrm{N}_{2} \mathrm{O}$ chemistry was controlled to vary the relative concentration of $\mathrm{Si}, \mathrm{O}$ and $\mathrm{N}$ [7]. The growth parameters and layer thickness are shown in Table 1.

The refractive index and thickness were measured by spectral reflectometry using a SCI Filmtek 4000 with a spectral range of 440 to $1660 \mathrm{~nm}$ at normal and $70^{\circ}$ incidence. The stoichiometry was determined by Rutherford 
Table 2: Experimental results of the stoichiometry measurements of the silicon oxynitride samples used in this study. The second and third columns express the relative presence of $\mathrm{SiO}_{2}$ and $\mathrm{Si}_{3} \mathrm{~N}_{4}$ in the samples. The information presented in the last four columns is a comparison of the $\mathrm{O}$ and $\mathrm{N}$ concentration, normalized to $\mathrm{Si}$, according to both techniques used in to characterised the silicon oxynitride layers.

\begin{tabular}{c|cccc|c|cc} 
Sample & \multicolumn{3}{|c}{ Spectral Reflectometry } & \multicolumn{2}{c}{ RBS } \\
$\mathrm{SiO}_{x} \mathrm{~N}_{y}$ & $\mathrm{X}_{\mathrm{SiO}_{2}}$ & $\mathrm{X}_{\mathrm{Si}_{3} \mathrm{~N}_{4}}$ & $x$ & $y$ & $x$ & $y$ \\
\hline & & & & & & \\
$\# 1$ & 0.608 & 0.392 & 1.09 & 0.61 & 1.15 & 0.45 \\
$\# 2$ & 0.827 & 0.173 & 1.53 & 0.28 & 1.32 & 0.37 \\
$\# 3$ & 0.948 & 0.052 & 1.82 & 0.08 & 1.67 & 0.14
\end{tabular}

backscattering spectrometry (RBS) with $2.4 \mathrm{MeV} \mathrm{He}^{+}$ ions.

Samples were irradiated with $185 \mathrm{MeV} \mathrm{Au}^{13+}$ ions at normal incidence and fluences of $1 \times 10^{11}$ and $3 \times 10^{11}$ ions $/ \mathrm{cm}^{2}$ to minimize track overlap effects. The SHI irradiation was performed at the Heavy Ion Acceletator Facility (HIAF) at the Australian National University.

Synchrotron SAXS measurements were performed at the SAXS/WAXS beamline at the Australian Synchrotron, using X-ray energies of $11 \mathrm{keV}$. Samples were prepared by reducing the thickness of the $\mathrm{Si}$ substrate to less than $100 \mu \mathrm{m}$ by mechanical polishing to reduce parasitic scattering. The experiments were performed with the $\mathrm{X}$-ray beam parallel to the ion tracks and tilted by $5^{\circ}$ and $10^{\circ}$ off the normal.

\section{Stoichiometry}

The refractive index of silicon oxynitride can be varied continuously according to the composition between the values of the end members $\left(n_{\mathrm{SiO}_{2}}=1.49\right.$ to $\left.n_{\mathrm{Si}_{3} N_{4}}=1.95\right)$. The relative composition of the silicon oxynitride can be calculated following the approximation of their effective refractive index:

$$
\begin{aligned}
& n_{\mathrm{SiO}_{x} \mathrm{~N}_{y}}=X_{\mathrm{SiO}_{2}} \cdot n_{\mathrm{SiO}_{2}}+X_{\mathrm{Si}_{3} \mathrm{~N}_{4}} \cdot n_{\mathrm{Si}_{3} \mathrm{~N}_{4}}, \\
& X_{\mathrm{SiO}_{2}}+X_{\mathrm{Si}_{3} \mathrm{~N}_{4}}=1 .
\end{aligned}
$$

From this relationship it is possible to calculate the relative concentration of $\mathrm{SiO}_{2}$ and $\mathrm{Si}_{3} \mathrm{~N}_{4}$. The results for the compositions used in this study are listed in Table 2. As a result, the corresponding concentration of $\mathrm{Si}, \mathrm{O}$ and $\mathrm{N}$ is obtained and compared with RBS measurements [8, 9]. For practical purposes, we consider the average values given by both techniques. Besides this simplification, a good agreement is achieved confirming the gradient-index material hypothesis.

The following reaction underlies the deposition process:

$$
a \mathrm{SiH}_{4}+b \mathrm{NH}_{3} \longrightarrow \mathrm{Si}_{x} \mathrm{~N}_{y} \mathrm{H}_{z}+c \mathrm{H}_{2}
$$

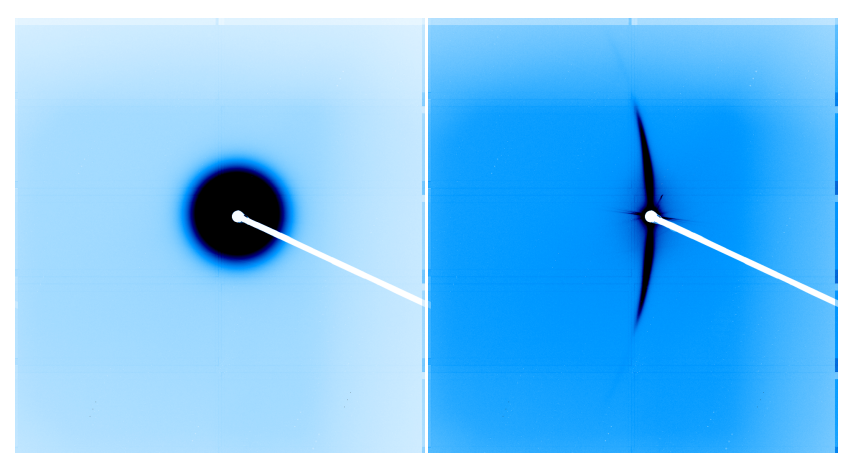

(a) $\sim 0^{\circ}$

(b) tilted $10^{\circ}$

Figure 1: Scattering images of ion tracks formed in $\mathrm{Si}_{3} \mathrm{~N}_{4}$ after being irradiated with $185 \mathrm{MeV}$ Au ions with a fluence of $1 \times 10^{11}$ ions $/ \mathrm{cm}^{2}$.

Silicon oxynitrides synthesized by PECVD techniques possess a high concentration of $\mathrm{H}$. At $600^{\circ} \mathrm{C}$, most of the $\mathrm{H}$ is released and the concentration decreases to $10 \%$ for $\mathrm{Si}_{3} \mathrm{~N}_{4}$, and to less than $3 \%$ for $\mathrm{SiO}_{2}$. The presence of $\mathrm{H}$ modifies the thermodynamical properties which can affect the track formation process, which will be studied in detail in the future.

\section{Ion Track Formation}

Silicon oxynitride samples were irradiated with $185 \mathrm{MeV}$ $\mathrm{Au}^{13+}$. At this energy, the electronic stopping power varies from $\sim 23 \mathrm{keV} / \mathrm{nm}$, in the case of $\mathrm{Si}_{3} \mathrm{~N}_{4}$, to $\sim 17 \mathrm{keV} / \mathrm{nm}$ for $\mathrm{SiO}_{2}$ [10] and can be considered constant trough the thickness of the layer.

In Figure 1, the SAXS images of ion tracks in $\mathrm{Si}_{3} \mathrm{~N}_{4}$ are presented Figure 1a correspond to the scattering pattern from ion tracks almost aligned to the X-ray beam. Concentrical interference rings can be observed due to the azimuthal symmetry present. As can be seen in Fig. 1b, when the sample is tilted $10^{\circ}$ from the X-ray beam two narrow curved streaks appear which confirm the high anisotropy of the ion tracks.

Figure 2 shows scattering spectra corresponding to the irradiated samples after removal of the corresponding background. The spectra were extracted from the intensities of the narrow streaks shown in Figure 1b. Due to the amorphous nature of our samples, it is valid to consider an azimuthal symmetry, in particular a cylindrical geometry.

The scattering intensity can be written as:

$$
I\left(q_{r}\right) \propto \int \exp \left(-\frac{R^{2}}{2 \sigma^{2}}\right)\left|f\left(q_{r}\right)\right|^{2} d R
$$

where the term $\left|f\left(q_{r}\right)\right|^{2}$ represents the scattering amplitude of the ion track, $R$ the total track radius and $\sigma$ the radius polydispersity.

The scattering intensity from our SAXS measurements can be extracted and fitted using a core-shell cylinder model (Figure 3). The model assumes a cylindrical symmetrical density distribution around the ion path with a 
Table 3: Data results by applying a core-shell model to the SAXS experimental data and covered area related to the overlapping percentage.

\begin{tabular}{|c|c|c|c|c|c|c|c|}
\hline & Fluence (ions $/ \mathrm{cm}^{2}$ ) & $\rho_{c} / \rho_{s}$ & Core $(\AA)$ & Shell $(\AA)$ & Radius $(\AA)$ & Covered area $(\%)$ & overlap (\%) \\
\hline \multirow[t]{2}{*}{$\mathrm{Si}_{3} \mathrm{~N}_{4}$} & $1 \times 10^{11}$ & $-8.4 \pm 0.4$ & $18 \pm 1$ & $24 \pm 1$ & $42 \pm 1$ & 5.6 & 0.2 \\
\hline & $3 \times 10^{11}$ & $-8.5 \pm 0.5$ & $18 \pm 1$ & $23 \pm 1$ & $41 \pm 1$ & 14.5 & 1.1 \\
\hline \multirow[t]{2}{*}{$\# 1-\mathrm{SiO}_{1.12} \mathrm{~N}_{0.53}$} & $1 \times 10^{11}$ & $-9.1 \pm 0.5$ & $24 \pm 1$ & $34 \pm 1$ & $58 \pm 1$ & 9.7 & 0.5 \\
\hline & $3 \times 10^{11}$ & $-9.6 \pm 0.7$ & $23 \pm 1$ & $33 \pm 1$ & $56 \pm 1$ & 25.9 & 4.0 \\
\hline \multirow[t]{2}{*}{$\# 2-\mathrm{SiO}_{1.43} \mathrm{~N}_{0.32}$} & $1 \times 10^{11}$ & $-5.9 \pm 0.3$ & $25 \pm 1$ & $32 \pm 1$ & $57 \pm 1$ & 9.5 & 0.5 \\
\hline & $3 \times 10^{11}$ & $-7.7 \pm 0.6$ & $24 \pm 1$ & $31 \pm 1$ & $55 \pm 1$ & 24.6 & 3.6 \\
\hline \multirow[t]{2}{*}{$\# 3-\mathrm{SiO}_{1.75} \mathrm{~N}_{0.11}$} & $1 \times 10^{11}$ & $-8.1 \pm 1.1$ & $23 \pm 1$ & $34 \pm 2$ & $57 \pm 2$ & 9.8 & 0.5 \\
\hline & $3 \times 10^{11}$ & $-7.7 \pm 0.6$ & $23 \pm 1$ & $32 \pm 1$ & $55 \pm 1$ & 22.8 & 3.1 \\
\hline \multirow[t]{2}{*}{$\mathrm{SiO}_{2}$} & $1 \times 10^{11}$ & $-4.9 \pm 0.2$ & $23 \pm 1$ & $30 \pm 1$ & $53 \pm 1$ & 8.6 & 0.4 \\
\hline & $3 \times 10^{11}$ & $-4.6 \pm 0.7$ & $23 \pm 1$ & $32 \pm 1$ & $55 \pm 1$ & 24.8 & 3.7 \\
\hline
\end{tabular}

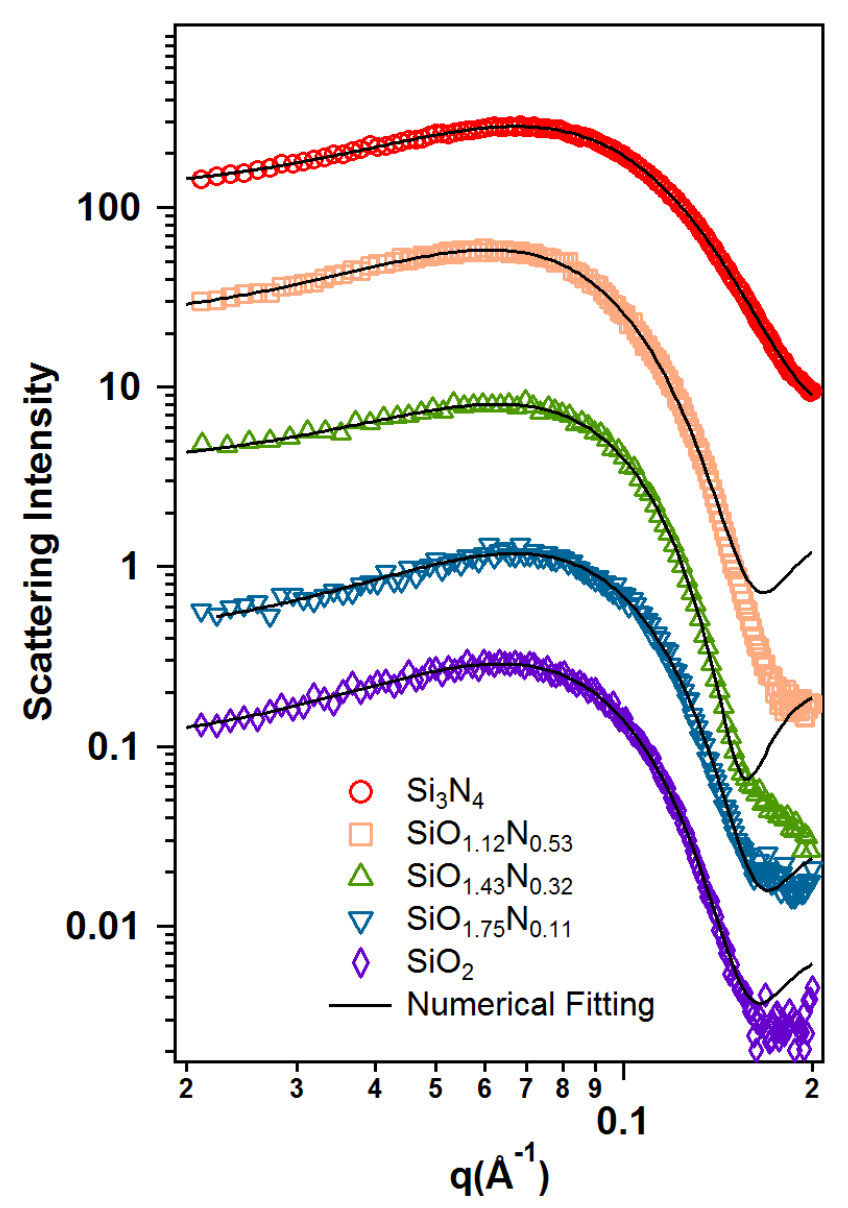

Figure 2: SAXS spectra of silicon oxynitrides irradiated with $185 \mathrm{MeV} \mathrm{Au}$ ions at with a fluence of $3 \times 10^{11}$ ions $/ \mathrm{cm}^{2}$. The solid lines represent the numerical fits to the core-shell model.

core and shell density different from the surrounding bulk material. The corresponding scattering amplitude $f\left(q_{r}\right)$

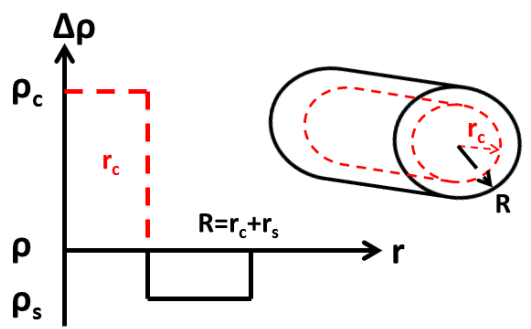

Figure 3: Form factor considered for the ion track structure in silicon oxynitride. The core shell-structure implies an overdense core and an underdense shell from the bulk material density.

can be derived as [11]:

$$
f\left(q_{r}\right)=\frac{2 \pi L}{q}\left[\left(1-\frac{\rho_{s}}{\rho_{c}}\right) R_{c} J_{1}\left(R_{c} q_{r}\right)+\frac{\rho_{s}}{\rho_{c}} R J_{1}(R q)\right],
$$

where $L$ is the track length (equivalent to the layer thickness), the terms $\rho_{c}$ and $\rho_{s}$ correspond to the density change of core and shell respectively, $\mathrm{R}$ is the total track radius, and $J_{1}$ the first-order Bessel function. The solid lines in Figure 2 show the fit to the data using this model.

The area covered by ion tracks can be calculated by: $d=1-\exp \left(-\pi R^{2} F\right)$, where $d$ is the modified area, $R$ is the total track radius and $F$ is the ion fluence. The area of track overlap is estimated as $\pi R^{2} F-d$. In Table 3 , the covered area and the overlapped fraction with respect to the covered area is listed. For the fluences of $1 \times 10^{11}$ and $3 \times 10^{11}$ ions $/ \mathrm{cm}^{2}$, the covered area is less than 10 and $25 \%$ respectively. The overlap remains below $5 \%$, the effects due to track overlapping can be neglected in our analysis.

Ion tracks in silicon oxynitride samples exhibited a total radius between 4 and $6 \mathrm{~nm}$. When normalized with the total track volume, a small fraction of the track volume belongs to the core (below $20 \%$ of the total volume) and a large fraction (above 80\%) resembles the shell volume for all the samples studied in this work. However, the density difference of the core with respect to the matrix exceeds 
that of the shell by a factor of 5-10. All the key results are listed in Table 3.

In the case of $\mathrm{Si}_{3} \mathrm{~N}_{4}$, ion tracks with an average total radius of $4.2 \pm 0.1 \mathrm{~nm}$ were observed, made-up of a core radius of $1.8 \pm 0.1 \mathrm{~nm}$ and a shell thickness of $2.4 \pm 0.1$ $\mathrm{nm}$. The relative scattering contrast of the core and shell was found to be: $\rho_{c} / \rho_{s}=-8.45 \pm 0.5$. This ratio compares the density change of the core and shell compared to the surrounding $\mathrm{Si}_{3} \mathrm{~N}_{4}$. The negative value of the ratio implies an overdense core and an underdense shell or vice versa, with respect to the matrix. Previously, molecular dynamics simulations in $\mathrm{SiO}_{2}$ showed an underdense core surrounded by an overdense shell [11].

For the silicon oxynitride samples \#1 to \#3, the total radius can be estimated to be $\sim 5.6 \pm 0.3 \mathrm{~nm}$ with a core radius of $2.3 \pm 0.2 \mathrm{~nm}$ and a shell thickness of $3.3 \pm 0.3$ $\mathrm{nm}$. As can be seen in Table 2, there is a significant variation in the N/O ratio among the three samples but the overall ion track dimensions are very similar. The only perceivable difference lies in the scattering contrast of the core and shell, this can be result of larger density differences between the core and the shell. For these samples, a slight decrease in the scattering contrast is achieved with a decrease of $\mathrm{N}$ concentration.

Track radii in $\mathrm{SiO}_{2}$ synthesized by PECVD can be directly compared to thermal a-SiO 2 [11]. A total radius of $5.4 \pm 0.2 \mathrm{~nm}$ with ion tracks exhibiting a core radius of $2.3 \pm 0.1 \mathrm{~nm}$ and a shell thickness of $3.1 \pm 0.1 \mathrm{~nm}$ were measured. A good agreement is achieved with a discrepancy of $30 \%$ of the ion track volume covered by the core and the shell, which can be related to the possible variation in density and microstructure of $\mathrm{a}-\mathrm{SiO}_{2}$ deposited by PECVD compared to thermal a- $\mathrm{SiO}_{2}$.

\section{Conclusions}

SAXS measurements performed on ion tracks formed by SHI irradiation of silicon oxynitride films demonstrate the presence of a core-shell structure with typical radius between 4 and $6 \mathrm{~nm}$. At low fluences it is possible to average a large amount of individual ion tracks without considerable overlap. In our case, silicon oxynitride films exhibit ion tracks with dimensions almost independent of the $\mathrm{N}$ concentration and larger than those in $\mathrm{Si}_{3} \mathrm{~N}_{4}$ and $\mathrm{SiO}_{2}$.

\section{Acknowledgments}

Authors would like to acknowledge the Consejo Nacional de Ciencia y Tecnologia. This work was performed at the SAXS/WAXS beamline at the Australian Synchrotron. P. K. and M. C. R. would like to thank the Australian Research Council for financial support.

\section{References}

[1] M. Waligorski, R. Hamm, R. Katz, International Journal of Radiation Applications and Instrumentation. Part D. Nuclear Tracks and Radiation Measurements 11, 309 (1986)

[2] M. Nastasi, J. Mayer, J. Hirvonen, Ion-Solid Interactions: Fundamentals and Applications, Cambridge Solid State Science Series (Cambridge University Press, 1996), ISBN 9780521373760

[3] K. Nakajima, Y. Morita, M. Suzuki, K. Narumi, Y. Saitoh, N. Ishikawa, K. Hojou, M. Tsujimoto, S. Isoda, K. Kimura, Nuclear Instruments and Methods in Physics Research Section B 291, 12 (2012)

[4] T. Bååk, Appl. Opt. 21, 1069 (1982)

[5] A. El amrani, I. Menous, L. Mahiou, R. Tadjine, A. Touati, A. Lefgoum, Renewable Energy 33, 2289 (2008)

[6] R. Huang, Z. Lin, Y. Guo, C. Song, X. Wang, H. Lin, L. Xu, J. Song, H. Li, Opt. Mater. Express 4, 205 (2014)

[7] F. Karouta, K. Vora, J. Tian, C. Jagadish, Journal of Physics D: Applied Physics 45, 445301 (2012)

[8] L.R. Doolittle, Nuclear Instruments and Methods in Physics Research Section B 9, 344 (1985)

[9] L.R. Doolittle, Nuclear Instruments and Methods in Physics Research B 15, 227 (1986)

[10] J.F. Ziegler, U. Littmark, J.P. Biersack, The stopping and range of ions in solids / J.F. Ziegler, J.P. Biersack, U. Littmark (Pergamon New York, 1985), ISBN 008021603

[11] P. Kluth, C. Schnohr, O. Pakarinen, F. Djurabekova, D. Sprouster, R. Giulian, M. Ridgway, A. Byrne, C. Trautmann, D. Cookson et al., Phys. Rev. Lett. 101, 175503 (2008) 